\title{
AMENDMENTS
}

\section{Author Correction: Ultralow-loss polaritons in isotopically pure boron nitride}

Alexander J. Giles, Siyuan Dai, Igor Vurgaftman, Timothy Hoffman, Song Liu, Lucas Lindsay, Chase T. Ellis, Nathanael Assefa, loannis Chatzakis, Thomas L. Reinecke, Joseph G. Tischler, Michael M. Fogler, J. H. Edgar, D. N. Basov and Joshua D. Caldwell

Correction to: Nature Materials https://doi.org/10.1038/nmat5047, published online 11 December 2017.

In the version of this Letter originally published, the isotopic enrichments in the caption of Fig. 1a were incorrectly given as "red${ }^{10} \mathrm{~B} 98.7 \%$, purple - natural abundant, brown $-{ }^{10} \mathrm{~B} 51 \%$, cyan $-{ }^{11} \mathrm{~B} 83 \%$ and blue $-{ }^{11} \mathrm{~B} 99.2 \%$ "; they should have read "red- $-{ }^{11} \mathrm{~B} 99.2 \%$, purple-natural abundant, brown $-{ }^{10} \mathrm{~B} 51 \%$, cyan $-{ }^{10} \mathrm{~B} 83 \%$ and blue- ${ }^{10} \mathrm{~B} 98.7 \%$.

Published online: 1 August 2019

https://doi.org/10.1038/s41563-019-0469-2

\section{Author Correction: Impacts of surface depletion on the plasmonic properties of doped semiconductor nanocrystals}

Omid Zandi (D), Ankit Agrawal, Alex B. Shearer, Lauren C. Reimnitz, Clayton J. Dahlman, Corey M. Staller and

Delia J. Milliron (D)

Correction to: Nature Materials https://doi.org/10.1038/s41563-018-0130-5, published online 9 July 2018.

In the originally published Supplementary Information file for this Article, on page 16 it incorrectly read "core radius of $a=r_{1}$ and shell outer radius of $b=r_{2}$ ", but should have read "core radius of $b=r_{1}$ and shell outer radius of $a=r_{2}$ ". The corrected Supplementary Information file has now been uploaded. 